\title{
The efficacy of levocarnitine treatment in relieving fatigue in patients with cirrhosis but without overt hepatic encephalopathy: a prospective non- randomized controlled study
}

Kazumichi Abe ( $\nabla$ k-abe@fmu.ac.jp)

Fukushima Medical University

Masashi Fujita

Department of Gastroenterology

Manabu Hayashi

Department of Gastroenterology

Atsushi Takahashi

Department of Gastroenterology

Hiromasa Ohira

Department of Gastroenterology

\section{Research}

Keywords: liver cirrhosis, fatigue, levocarnitine, thioredoxin

Posted Date: May 8th, 2020

DOI: https://doi.org/10.21203/rs.3.rs-26504/v1

License: (c) (i) This work is licensed under a Creative Commons Attribution 4.0 International License.

Read Full License 


\section{Abstract}

Background In recent years, levocarnitine has been reported to be an effective treatment in patients with liver cirrhosis, but its mechanism is not fully understood. In the present study, we prospectively examined the efficacy of levocarnitine in relieving symptoms of fatigue in patients with cirrhosis but without overt hepatic encephalopathy.

Methods Twenty-one cirrhotic patients who were able to undergo fatigue symptom evaluations at our institution were enrolled. A total of 12 cirrhotic patients underwent levocarnitine treatment (1200-1800 $\mathrm{mg} /$ day), and 9 patients did not undergo levocarnitine treatment. Patient age, sex, and Child-Pugh (C-P) class, complications and the etiology of cirrhosis were assessed. As primary end-points, we investigated whether the levocarnitine treatment exerted any beneficial effects by assessing the symptoms of fatigue [8-item Short-Form Health Survey (SF-8) and Fisk Fatigue Severity Scores (FFSSs)] at baseline and 3 months after treatment. Furthermore, as exploratory secondary end-points, we investigated whether the levocarnitine treatment exerted effects on ameliorating oxidative stress by assessing the serum thioredoxin (TRX) and urinary 8-hydroxydeoxyguanosine (8-OhdG) levels.

Results The median age of the patients was 73 years. Three males and 18 females had C-P classes A and $B$ ( 14 and 7 patients, respectively), and 3 patients (14.3\%) had a history of hepatocellular carcinoma treatment. A total of 6 cases of diabetes (28.6\%), 6 cases of ascites (28.6\%), and 4 cases of varices $(19.0 \%)$ were identified. There were no significant differences in the clinical laboratory values between the two groups. The FFSS and SF-8 scores were significantly improved in the patients with cirrhosis who underwent levocarnitine treatment $(P<0.01)$ but not in patients who did not undergo levocarnitine treatment. Furthermore, three months after the levocarnitine treatment, the serum carnitine concentrations were significantly increased and the serum thioredoxin levels were decreased in the patients with cirrhosis who underwent levocarnitine treatment $(P<0.005)$.

Conclusion These results suggest that levocarnitine treatment may relieve symptoms of fatigue in cirrhotic patients by reducing oxidative stress.

\section{Background}

Fatigue, which is characterized as a persistent sense of exhaustion that prevents individuals from performing usual tasks and decreases their capacity for physical and mental work, is one of the most common symptoms reported by patients in general medical practice [1, 2]. Fatigue is also considered to be common in individuals with chronic liver disease (CLD). However, because it is difficult to define and treat fatigue, this symptom is often overlooked or minimized by physicians caring for patients with CLD. Although reports on fatigue in patients with cholestatic liver disease [3-5], chronic hepatitis C [6,7], and nonalcoholic fatty liver disease [8] have been published, few of the patients in these studies were diagnosed with overt cirrhosis. Thus, published data on fatigue and its possible association with health- 
related quality of life (HRQOL) in individuals with cirrhosis are scarce, and our understanding of fatigue in patients with cirrhosis remains limited [9].

A useful construct is to consider fatigue as two separate entities: peripheral fatigue and central fatigue [10]. Peripheral fatigue results from neuromuscular dysfunction originating from noncentral nervous system mechanisms and most commonly manifests as weakness in clinical examinations [11-13]. Although changes in muscle metabolism have been identified in patients with CLD, peripheral fatigue in general appears to be of lesser importance in patients with liver disease, and cirrhosis and liver failure are often associated with muscle wasting and sarcopenia [11]. Central fatigue results from altered neurotransmission within the brain and is often closely associated with other neuropsychiatric complaints that are presumed to be secondary to altered central neurotransmission, namely, depression and anxiety $[11,14,15]$.

Regarding fatigue assessment tools, the Fatigue Impact Score (FIS) has been validated for patient selfcompletion and is widely used $[16,17]$. In a study investigating the effect of fatigue on the QOL and mental health status of patients with primary biliary cirrhosis (PBC), the mean FIS was significantly increased compared to healthy controls [18-20]. In another study evaluating the effects of fatigue on sleep, depression, and liver disease severity in patients with PBC, the self-rated Fatigue Severity Score (FSS) correlated well with the verbally reported severity of fatigue [21]. The Fisk Fatigue Severity Score (FFSS) is also a highly acceptable, internally consistent and reproducible measure of fatigue severity in individuals with PBC [22]. For global function and QOL scores, measures such as the 36- or 8-item ShortForm Health Survey (SF-36 and SF-8) have been used and, perhaps unsurprisingly, show that fatigue is one of the major contributing factors to life quality impairment in individuals with CLD. Furthermore, global function can be very poor in individuals with CLD [23-25]. In a study that used the SF-8 to evaluate HRQOL in 300 patients with compensated cirrhosis, the median SF-8 score was 70 (interquartile range [IQR], 54-86) [26].

On the other hand, a previous study revealed an important role for oxidative stress in chronic fatigue [27]. Biomarkers of oxidative stress are defined as biological molecules whose chemical structures have been modified by free radicals [28]. The thioredoxin (TRX) system exists in all living cells and, according to its evolutionary history, is considered to comprise genetic material linked to DNA and to serve as a defense against oxidative damage. Elevated serum TRX levels have been associated with many diseases, including diabetes, chronic kidney disease, severe brain injury, coronary disease and hepatocellular carcinoma [29-33]. Furthermore, oxidative stress-related urinary biomarkers, such as 8hydroxydeoxyguanosine (8-OHdG), have been used to assess psychological distress. The urinary 8-OHdG level is a putative biomarker of total oxidative stress.

Levocarnitine transports long-chain fatty acids from the cytosol into the mitochondrial matrix for subsequent $\beta$-oxidation. According to previous studies, levocarnitine supplementation exerts positive effects on oxidative stress, inflammation, fatigue, QOL, nutritional status, and sarcopenia [34]. 
The aim of this study was to evaluate the efficacy of exogenous levocarnitine in relieving physical and mental fatigue in patients with cirrhosis but without overt hepatic encephalopathy.

\section{Patients And Methods \\ Study design}

A prospective non-randomized controlled study was conducted at Fukushima Medical University (Fukushima, Japan) between February 2015 and January 2018. We prospectively evaluated the symptoms of fatigue in 21 patients with liver cirrhosis undergoing levocarnitine treatment using the SF-8 and FFSS. A dose of levocarnitine (1200 mg or $1800 \mathrm{mg}$ ) was given orally three times a day for 3 months to 12 patients. A total of 9 of the 21 patients were not given levocarnitine. The protocol was conducted with the approval of the institutional review board of our institution and in accordance with the World Medical Association's Declaration of Helsinki. The final protocol was approved by the Ethics Committee of Fukushima Medical University in Fukushima, Japan (No. 1501). Written informed consent was obtained from all patients. Liver cirrhosis was diagnosed based on the presence of morphologic changes in the liver, such as hypertrophy of the left lateral and caudate lobes or atrophy of the right posterior haptic lobe, as identified by ultrasonography, computed tomography (CT), and/or magnetic resonance imaging (MRI), or the presence of pseudolobule formation, as identified by a histopathologic examination, or the presence of portal hypertension, such as varices. Patients with severe liver dysfunction, such as decompensated cirrhosis with C-P score $\geq 10$ or residual hepatocellular carcinoma (HCC), were excluded. The patients with cirrhosis presenting with overt hepatic encephalopathy were excluded. Overt hepatic encephalopathy was diagnosed according to the following criteria: 1) disturbed consciousness in the absence of other causes, such as intracranial disease, serious systemic infection, hypoglycemia, electrolyte disorders, and drug or alcohol abuse; 2) a blood ammonia concentration greater than the baseline value; and 3) the presence of asterixis.

\section{Instruments for QOL assessment}

A disease-specific HRQOL analysis and a cross-sectional analysis of general HRQOL were conducted using the Japanese version of a questionnaire on subjective and objective symptoms in patients with liver cirrhosis and using the Japanese version of the Medical Outcomes Study SF-8. The validity and reliability of the Japanese versions of both questionnaires have been confirmed, as described previously [35]. The Japanese version of the questionnaire on subjective and objective symptoms comprises 6 subscales (sluggishness, fatigue, general itching, anorexia, abdominal fullness, and muscle cramps). The SF-8 comprises 8 subscales (general health, GH; physical functioning, $\mathrm{PH}$; role limitation due to physical problems, RP; body pain, BP; vitality, VT; social functioning, SF; mental health, $\mathrm{MH}$; role limitation due to emotional problems, RE), and all of these categories are consistent with those in the SF-8. In the present study, the scores for each of the 8 subscales, the physical health component summary score (PCS), and the mental health component summary score (MCS) were measured using the norm-based scoring method, which was based on a large-scale population study conducted in Japan [35]. 
Fatigue was assessed using the Japanese version of the FFSS [2]. The FFSS includes three subscales to assess the perceived impact of fatigue on cognitive functioning (10 items), physical functioning (10 items), and psychosocial functioning (20 items). The FFSS is a self-report instrument, for which subjects are asked to rate the extent to which fatigue has caused problems for them in relation to example statements $(0=$ no problems to $4=$ extreme problems, maximum FFSS $=160)$.

\section{Clinical and laboratory assessments}

Detailed clinical and demographic information, including the patient's age, sex, and complications [diabetes mellitus (DM), varices, ascites, shunt, and HCC] and etiology of cirrhosis were collected. Laboratory data, including the aspartate aminotransferase (AST), alanine aminotransferase (ALT), albumin, total bilirubin, hemoglobin, and $\mathrm{NH}_{3}$ levels; prothrombin time; platelet (PLT) count and serum levels of carnitine fractions (total carnitine, free carnitine, and acylcarnitine), were evaluated before and 3 months after levocarnitine treatment. DM was defined as the presence of any of the following criteria: (i) a documented history of diabetes, (ii) the consumption of a diabetes medication, or (iii) a fasting glucose level of $\geq 126 \mathrm{mg} / \mathrm{dl}$ or HgbA1C level of $\geq 6.5$ on two separate occasions.

\section{Thioredoxin and 8-OhdG assessments}

The level of TRX was measured in serum samples, and 8-OhdG was measured in urine samples with enzyme-linked immunosorbent assay (ELISA) kits in accordance with the manufacturer's instructions. The following kits were used for all measurements: the serum TRX (Human TRX ELISA kit, CUSABIO, Hubei, China) and urinary 8-OhdG kits (New 8-OHdG Check; Nikken Zeil, Fukuroi, Shizuoka, Japan).

\section{Primary and secondary end-points}

As primary end-points, we investigated whether the levocarnitine treatment exerted any beneficial effects on the symptoms of fatigue (SF-8 and FFSS) at baseline and 3 months after treatment.

As exploratory secondary end-points, we investigated whether the levocarnitine treatment exerted effects on ameliorating oxidative stress by measuring the serum TRX and urinary 8-OhdG levels.

\section{Statistical analysis}

Continuous variables were described as median (interquartile range [IQR]). Differences were compared using the Mann-Whitney $\mathrm{U}$ test and Wilcoxon matched-pairs signed-rank test. Correlations between variables were assessed using Spearman's rank correlation coefficient. A multivariate logistic regression analysis was performed to analyze the factors related to the decrease in the serum TRX levels. All statistical analyses were performed using Prism 6.0 software (GraphPad Software, Inc.) and JMP Pro 13.1 (SAS Institute Inc., Cary, NC). P $<0.05$ indicated statistical significance.

\section{Results}


The patients' baseline characteristics are shown in Table 1. A total of 3 male and 18 female patients were enrolled. The median age of the patients at the start of treatment was 73 years. The etiology of cirrhosis was the hepatitis $\mathrm{C}$ virus in 10 patients, nonalcoholic steatohepatitis in 4 patients, primary biliary cholangitis in 3 patients, alcoholic liver dysfunction in 2 patients, and other causes in 2 patients. The Child-Pugh classification was A in 14 cases and B in 7 cases. A total of 3 patients had previously undergone HCC treatment (14.3\%), 6 patients had DM (28.6\%), 6 patients had ascites (28.6\%), 4 patients had varices (19.0\%), and 4 patients had shunts (14.3\%). No patients had muscle cramps during this study.

The laboratory data recorded for patients with cirrhosis before and after levocarnitine treatment are compared in Table 2. No significant differences in the clinical laboratory results, including the ammonia and urinary 8-OhdG levels, were observed before and after levocarnitine treatment in either group. Although the serum levels of carnitine fractions in the patients with cirrhosis who did not undergo levocarnitine treatment were within the standard ranges, these levels were significantly increased in the patients with cirrhosis who underwent levocarnitine treatment.

Clinical effects of levocarnitine treatment on symptoms of fatigue in cirrhotic patients

Significant differences in mental HRQOL, as measured by the SF-8 MCS, were not observed between the patients with cirrhosis treated with or without levocarnitine when assessed before and 3 months after levocarnitine treatment (patients who underwent levocarnitine treatment; $P=0.8501$, patients who did not undergo levocarnitine treatment; $P=0.8438$ ) (Figure 1A and B). Conversely, patients with cirrhosis who underwent levocarnitine treatment were more likely to report a significantly impaired physical HRQOL, as measured by the SF-8 PCS (patients who underwent levocarnitine treatment; $P=0.0068$, patients who did not undergo levocarnitine treatment; $P=0.3828$ ) (Figure $1 \mathrm{C}$ and $\mathrm{D}$ ).

Furthermore, the FFSS for fatigue was significantly improved in patients who underwent levocarnitine treatment $(P=0.0068)$, but did not improve in patients who did not undergo levocarnitine treatment $(P=0.4922)$ (Figure $2 \mathrm{~A}$ and $\mathrm{B})$.

\section{Levocarnitine treatment reduces serum thioredoxin levels in patients with cirrhosis}

In patients who underwent levocarnitine treatment, we observed a significant decrease in serum TRX levels (Figure 3A, B). Moreover, in the comparison between cirrhotic patients who did and did not undergo levocarnitine treatment, significant differences in the $\Delta$ serum TRX levels [(serum TRX levels at posttreatment) - (serum TRX levels at pretreatment)] were observed ( $P=0.0013$ ) (Figure 3C). In contrast, there were no significant differences in the urinary 8-OhdG levels, which is thought to be an oxidative stress marker, in the cirrhotic patients who underwent levocarnitine treatment. 
Furthermore, the Spearman's rank coefficient analysis results are shown in Figure 4. Although the $\triangle$ serum TRX levels did not correlate with the serum acylcarnitine concentration after 3 months, they were significantly negatively correlated with the serum total carnitine and free carnitine concentrations after 3 months in the cirrhotic patients (total carnitine; $r=-0.4948, P=0.0226$, free carnitine; $r=-0.4831, P=0.0265$ ). The results of the multivariate logistic regression analysis of the factors significantly associated with the decrease in serum TRX levels in patients with cirrhosis are shown in Table 3. According to the Receiver Operating Characteristic (ROC) value for the decrease in the serum TRX levels based on free carnitine concentrations after 3 months observed in 21 patients with cirrhosis (sensitivity, 75.00\%; specificity, 88.89\%; AUC, 0.8056), the cut-off value for free carnitine concentrations after 3 months was set to 61.5 $\mu \mathrm{mol} / \mathrm{L}$. After adjusting for confounding variables, such as age, sex, and $\mathrm{NH}_{3}$ levels, free carnitine concentrations $>61.5 \mu \mathrm{mol} / \mathrm{L}$ after 3 months were an independent factor contributing to the decrease in serum TRX levels.

\section{Discussion}

In the present study, we investigated the efficacy of levocarnitine treatment in relieving symptoms of fatigue in patients with cirrhosis. Levocarnitine is critical for mitochondrial fatty acid oxidation, and levocarnitine supplementation has been shown to improve QOL and relieve symptoms of fatigue in patients with chronic kidney disease, cancer, and chronic hepatitis [36-39]. To the best of our knowledge, this report is the first to show that a levocarnitine treatment improves the physical symptoms of fatigue in patients with cirrhosis but without overt hepatic encephalopathy by reducing oxidative stress.

Furthermore, $\Delta$ serum TRX levels were significantly negatively correlated with serum total carnitine and free carnitine concentrations 3 months after levocarnitine treatment in cirrhotic patients. As fatigue is considered to be common in patients with cirrhosis, we believe that the results of our study have important implications for these patients.

Several reports have described the effects of levocarnitine supplementation on hepatic encephalopathy and muscle cramps in patients with cirrhosis [40-44]. According to a previous study, patients with cirrhosis presenting with hepatic encephalopathy who were treated with acetyl-levocarnitine showed a decrease in the severity of both mental and physical fatigue and an increase in physical activity [45]. Furthermore, a significant decrease in serum ammonia concentrations and a significant improvement in mental function in patients treated with acetyl-levocarnitine were observed. Acetyl-levocarnitine is an endogenous molecule synthesized in mitochondria by the enzyme acetyl-levocarnitine transferase and is the predominant type of acylcarnitine in normal tissue. The relationship between serum acylcarnitine and free carnitine is highly sensitive to intramitochondrial metabolic changes. In the present study, there were no significant differences in mental HRQOL, as measured by the SF-8 MCS, in cirrhotic patients who did and did not undergo levocarnitine treatment from baseline to 3 months after levocarnitine treatment. The cirrhotic patients in the present study did not have hepatic encephalopathy before they underwent levocarnitine treatment, and there were no significant differences in ammonia levels from baseline to 3 months after levocarnitine treatment. 
Under normal conditions, $80 \%$ of the total serum carnitine is free carnitine, and $20 \%$ is acylcarnitine, with a normal acylcarnitine-to-free carnitine ratio of $0.25[46,47]$. In the present study, although the serum total carnitine, free carnitine, and acylcarnitine concentrations were all within the standard ranges in the majority of the cirrhotic patients, these concentrations significantly increased in the patients who underwent levocarnitine treatment.

There is evidence from both animal and clinical studies showing that levocarnitine supplementation improves the balance of nitrogen either due to increased protein synthesis or reduced protein degradation, an inhibition of apoptosis and an abrogation of inflammatory processes under pathologic conditions. Furthermore, animal studies have provided strong evidence that levocarnitine supplementation prevents oxidative stress and ameliorates mitochondrial dysfunction [48]. TRX is a small and versatile protein that functions as a free radical scavenger to eliminate hydrogen peroxide and protect cells from oxidative injury [49]. Elevated serum TRX levels have been associated with many diseases, including diabetes, chronic kidney disease, severe brain injury, and coronary disease [29-32]. Levocarnitine protects cells from oxidative damage because it functions as a free radical scavenger and in lipid metabolism by transporting fatty acids across the inner mitochondrial membrane [50]. In the present study, we observed a significant decrease in serum TRX levels in cirrhotic patients who underwent levocarnitine treatment. Furthermore, the $\Delta$ serum TRX levels were significantly negatively correlated with serum total carnitine and free carnitine concentrations after 3 months in the cirrhotic patients. Free carnitine concentrations > $61.5 \mu \mathrm{mol} / \mathrm{L}$ after 3 months were an independent factor contributing to the decrease in serum TRX levels. The dose of levocarnitine was not significantly associated with percentages of patients with relieving symptoms of fatigue and an amelioration of serum TRX levels after 3 months (data not shown). Urinary $8-O H d G$ levels are a putative biomarker of total systemic oxidative stress, and psychological distress is associated with oxidative stress. In the present study, there were no significant differences in urinary 8OhdG levels or mental HRQOL in the cirrhotic patients who underwent levocarnitine treatment.

Our study has several limitations. First, the sample population was relatively small. Second, we measured TRX levels in serum, not in histologic specimens. It is still unclear whether serum and peripheral TRX levels reflect similar changes in the liver and muscle tissue. The relationship between peripheral and tissue TRX levels should be investigated further. Third, our intervention study conducted with patients with cirrhosis did not include a randomized design. Additional studies are required to increase the level of evidence.

\section{Conclusion}

Collectively, levocarnitine produced potentially favorable effects on symptoms of fatigue after 3 months of treatment in cirrhotic patients, by reducing oxidative stress in particular. Additional clinical studies with levocarnitine need to be conducted to determine the mechanism by which levocarnitine relieves fatigue.

\section{Abbreviations}


8-OHdG: 8-hydroxydeoxyguanosine; ALT: alanine aminotransferase; AST: aspartate aminotransferase; CLD: chronic liver disease; C-P: Child-Pugh; CT: computed tomography; DM: diabetes mellitus; ELISA: enzyme-linked immunosorbent assay; FSS: Fatigue Severity Score; FFSS: Fisk Fatigue Severity Score; FIS: Fatigue Impact Score; HCC: hepatocellular carcinoma; HRQOL: health-related quality of life; IQR: interquartile range; MCS: mental health component summary score; MRI: magnetic resonance imaging; PBC: primary biliary cirrhosis; PCS: physical health component summary score; PLT: platelet; SF-8: 8-item Short-Form Health Survey; SF-36: 36-item Short-Form Health Survey; TRX: thioredoxin.

\section{Declarations}

\section{Ethics approval and consent to participate}

The protocol was conducted with the approval of the institutional review board of our institution and in accordance with the World Medical Association's Declaration of Helsinki. The final protocol was approved by the Ethics Committee of Fukushima Medical University in Fukushima, Japan (No. 1501). Written informed consent was obtained from all patients.

\section{Consent for publication}

Not applicable.

\section{Competing interests}

The authors declare that they have no competing interests.

\section{Funding}

This study did not receive any funding body for its design, analysis and interpretation of data, or for writing the manuscript.

\section{Authors' contributions}

K.A. and H.O. designed the study and analyzed the patient data. K.A. drafted the manuscript. M.F., M.H., and A.T. collected and analyzed data. H.O. critically revised the manuscript for important intellectual content. All authors were involved in interpreting the data and have read and approved the final version of the manuscript. 


\section{Acknowledgments}

We thank Chikako Saito and Rie Hikichi for their technical assistance.

\section{Availability of data and material}

All data generated or analyzed during this study are included in this published article.

\section{References}

1. Kroenke K, Wood DR, Mangelsdorff DA, Meirer NJ, Powell JB. Chronic fatigue in primary care: prevalence, patient characteristics, and outcome. JAMA. 1988;260:929-34.

2. Fisk JD, Ritvo PG, Ross L, Haase DA, Marrie TJ, Schlech WF. Measuring the Functional Impact of Fatigue: Initial Validation of the Fatigue Impact Scale. Clin Infect Dis. 1994 Jan;18(Suppl 1):79-83.

3. Huet PM, Deslauriers J, Tran A, et al. Impact of fatigue on the quality of life of patients with primary biliary cirrhosis. Am J Gastroenterol. 2000;95:760-7.

4. Goldblatt J, Taylor PJ, Lipman T, et al. The true impact of fatigue in primary biliary cirrhosis: a population study. Gastroenterology. 2002;122:1235-41.

5. Björnsson E, Simren M, Olsson R, et al. Fatigue in patients with primary sclerosing cholangitis. Scand J Gastroenterol. 2004;39:961-8.

6. Barkhuizen A, Rosen HR, Wolf $S$, et al. Musculoskeletal pain and fatigue are associated with chronic hepatitis C: a report of 239 hepatology clinic patients. Am J Gastroenterol. 1999;94:1355-60.

7. McDonald J, Jayasuriya J, Bindley P, et al. Fatigue and psychological disorders in chronic hepatitis C. J Gastroenterol Hepatol. 2002;17:171-6.

8. Newton JL, Jones DE, Henderson E, et al. Fatigue in non-alcoholic fatty liver disease (NAFLD) is significant and associates with inactivity and excessive daytime sleepiness but not with liver disease severity or insulin resistance. Gut. 2008;57:807-13.

9. Kalaitzakis E, Josefsson A, Castedal M, Henfridsson P, Bengtsson M, Hugosson I, Andersson B, Björnsson E. Factors related to fatigue in patients with cirrhosis before and after liver transplantation. Clin Gastroenterol Hepatol. 2012 Feb;10(2):174-81.

10. Swain MG, Jones DEJ. Fatigue in Chronic Liver Disease: New Insights and Therapeutic Approaches. Liver Int. 2019 Jan;39(1):6-19.

11. Swain MG. Fatigue in liver disease: pathophysiology and clinical management. Can J Gastroenterol. 2006;20(3):181-8.

12. Austin PW, Gerber L, Karrar AK. Fatigue in chronic liver disease: exploring the role of the autonomic nervous system. Liver Int. 2015;35(5):1489-91. 
13. Stinton L, Swain MG. Fatigue in cirrhosis: is transplant the answer? Clin Gastroenterol Hepatol. 2012;10(2):103-5.

14. D'Mello C, Swain MG. Liver-brain interactions in inflammatory liver diseases: implications for fatigue and mood disorders. Brain Behav Immun. 2014;35:9-20.

15. D'Mello C, Swain MG. Liver-brain inflammation axis. Am J Physiol Gastrointest Liver Physiol. 2011;301(5):G749-61.

16. Prince MI, James OFW, Holland NP, Jones DEJ. Validation of a fatigue impact score in primary biliary cirrhosis: towards a standard for clinical and trial use. J Hepatol. 2000;32:368-73.

17. Dyson JK, Elsharkawy AM, Lamb CA, et al. Fatigue in primary sclerosing cholangitis is associated with sympathetic over-activity and increased cardiac output. Liver Int. 2015;35:1633-41.

18. Theal JJ, Toosi MN, Girlan L, Heslegrave RJ, Huet PM, Burak KW, Swain M, Tomlinson GA, Heathcote EJ. A randomized, controlled crossover trial of ondansetron in patients with primary biliary cirrhosis and fatigue. Hepatology. 2005 Jun;41(6):1305-12.

19. Jones DE, Bhala N, Burt J, Goldblatt J, Prince M, Newton JL. Four year follow up of fatigue in a geographically defined primary biliary cirrhosis patient cohort. Gut. 2006 Apr;55(4):536-41.

20. Newton JL, Gibson GJ, Tomlinson M, Wilton K, Jones D. Fatigue in primary biliary cirrhosis is associated with excessive daytime somnolence. Hepatology. 2006;44:91-8.

21. Cauch-Dudek K, Abbey S, Stewart DE, Heathcote EJ. Fatigue in primary biliary cirrhosis. Gut. 1998 Nov;43(5):705-10.

22. Prince MI, James OF, Holland NP, Jones DE. Validation of a fatigue impact score in primary biliary cirrhosis: towards a standard for clinical and trial use. J Hepatol. 2000 Mar;32(3):368-73.

23. Elliott C, Frith J, Day CP, et al. Functional impairment in alco- holic liver disease and non-alcoholic fatty liver disease is sig- nificant and persists over 3 years of follow-up. Dig Dis Sci. 2013;58(8):2383-91.

24. Elliott C, Frith J, Pairman J, et al. Reduction in functional ability is significant post-liver transplantation compared with matched liver disease and community dwelling controls. Transplant Int. 2011;24:588-95.

25. Newton JL, Elliott C, Frith J, et al. Functional capacity is signifi- cantly impaired in primary biliary cirrhosis and related to ortho- static symptoms. Eur J Gastroenterol Hepatol. 2011;23:566-72.

26. Tapper EB, Baki J, Parikh ND, Lok AS. Frailty. Psychoactive Medications, and Cognitive Dysfunction Are Associated With Poor Patient-Reported Outcomes in Cirrhosis. Hepatology. 2019 Apr;69(4):167685.

27. Lee JS, Kim HG, Lee DS, Son CG. Oxidative Stress Is a Convincing Contributor to Idiopathic Chronic Fatigue Sci Rep. 2018 Aug 27;8(1):12890.

28. Offord E, van Poppel G, Tyrrell R. Markers of Oxidative Damage and Antioxidant Protection: Current Status and Relevance to Disease. Free Radic Res. 2000 Nov;33 Suppl:5-19. 
29. Kakisaka Y, Nakashima T, Sumida Y, Yoh T, Nakamura H, Yodoi J, Senmaru H. Elevation of serum thioredoxin levels in patients with type 2 diabetes. Hormone metabolic research. 2002;34:160-4.

30. Tsuchikura S, Shoji T, Shimomura N, Kakiya R, Emoto M, Koyama H, Ishimura E, Inaba M, Nishizawa Y. Serum C-reactive protein and thioredoxin levels in subjects with mildly reduced glomerular filtration rate BMC nephrology. 2010; 11:7.

31. Abdiu A, Nakamura H, Sahaf B, Yodoi J, Holmgren A, Rosén A. Thioredoxin blood level increases after severe burn injury. Antioxidants redox signaling. 2000;2:707-16.

32. Miyamoto S, Kawano H, Sakamoto T, Soejima H, Kajiwara I, Hokamaki J, Hirai N, Sugiyama S, Yoshimura M, Yasue H, Nakamura H, Yodoi J, Ogawa H. Increased plasma levels of thioredoxin in patients with coronary spastic angina. Antioxidants Redox Signaling. 2004;6:75-80.

33. Li J, Cheng ZJ, Liu Y, Yan ZL, Wang K, Wu D, Wan XY, Xia Y, Lau WY, Wu MC, Shen F. Serum Thioredoxin Is a Diagnostic Marker for Hepatocellular Carcinoma. Oncotarget. 2015 Apr 20;6(11):9551-63.

34. Malaguarnera M, Cammalleri L, Gargante MP, Vacante M, Colonna V, Motta M. L-Carnitine Treatment Reduces Severity of Physical and Mental Fatigue and Increases Cognitive Functions in Centenarians: A Randomized and Controlled Clinical Trial. Am J Clin Nutr. 2007 Dec;86(6):1738-44.

35. Fukuhara S, Suzukamo Y. Manual of the SF-8 Japanese version. Kyoto: Institute for Health Outcome and Process Evaluation Research; 2004.

36. Duranay M, Akay H, Yilmaz FM, Senes M, Tekeli N, et al. Effects of L-carnitine infusions on inflammatory and nutritional markers in haemodialysis patients. Nephrol Dial Transplant. 2006;21:3211-4.

37. Biolo G, Stulle M, Bianco F, Mengozzi G, Barazzoni R, et al. Insulin action on glucose and pro- tein metabolism during L-carnitine supplementation in maintenance haemodialysis patients. Nephrol Dial Transplant. 2008;23:991-7.

38. Mantovani G, Maccio A, Madeddu C, Gramignano G, Serpe R, et al. Randomized phase III clinical trial of five different arms of treatment for patients with cancer cachexia: interim results. Nutrition. 2008;24:305-13.

39. Malaguarnera M, Vacante M, Bertino G, Neri S, Malaguarnera M, et al. The supplementation of acetylL-carnitine decreases fatigue and increases quality of life in patients with hepatitis $C$ treated with pegylated interferon-alpha 2b plus ribavirin. J Interferon Cytokine Res. 2011;31:653-9.

40. Malaguarnera M, Pistone G, Astuto M, et al. L-carnitine in the treatment of mild or moderate hepatic encephalopathy. Dig Dis. 2003;21:271-5.

41. Malaguarnera M, Pistone G, Elvira R, et al. Effects of L-carnitine in patients with hepatic encephalopathy. World J Gastroenterol. 2005;11:7197-202.

42. Malaguarnera M, Gargante MP, Cristaldi E, et al. Acetyl-L-carnitine treatment in minimal hepatic encephalopathy. Dig Dis Sci. 2008;53:3018-25.

43. Nakanishi $\mathrm{H}$, Kurosaki M, Tsuchiya $\mathrm{K}$, et al. L-carnitine reduces muscle cramps in patients with cirrhosis. Clin Gastroenterol Hepatol. 2015;13:1540-3. 
44. Shiraki M, Shimizu M, Moriwaki H, Okita K, Koike K. Carnitine dynamics and their effects on hyperammonemia in cirrhotic Japanese patients. Hepatol Res. 2017;47:321-7.

45. Malaguarnera M, Vacante M, Giordano M, Pennisi G, Bella R, Rampello L, Malaguarnera M, Li Volti G, Galvano F. Oral acetyl-L-carnitine Therapy Reduces Fatigue in Overt Hepatic Encephalopathy: A Randomized, Double-Blind, Placebo-Controlled Study. Am J Clin Nutr. 2011;93:799-808.

46. Campos Y, Huertas R, Lorenzo G, Bautista J, Gutié rrez E, Aparicio M, et al. Plasma carnitine insufficiency and effectiveness of L-carnitine therapy in patients with mitochondrial myopathy. Muscle Nerve. 1993;16:150-3.

47. Bohles H, Evangeliou A, Bervoets K, Eckert I, Sewell A. Carnitine esters in metabolic disease. Eur J Pediatr. 1994;153:57-61.

48. Ringseis R, Keller J, Eder K. Mechanisms underlying the anti-wasting effect of L-carnitine supplementation under pathologic conditions: evidence from experimental and clinical studies. Eur $\mathrm{J}$ Nutr. 2013;525:1421-42.

49. Sugama K, Suzuki K, Yoshitani K, Shiraishi K, Miura S, Yoshioka H, Mori Y, Kometani T. Changes of Thioredoxin, Oxidative Stress Markers, Inflammation and Muscle/Renal Damage Following Intensive Endurance Exercise. Exerc Immunol Rev. 2015;21:130-42.

50. Ribas GS, Vargas CR, Wajner M. L-carnitine supplementation as a potential antioxidant therapy for inherited neurometabolic disorders. Gene. 2014;533:469-76.

\section{Tables}

Table 1 Cirrhotic patients' baseline clinical and biochemical characteristics

\begin{tabular}{|c|c|c|c|}
\hline & $\begin{array}{l}\text { Without levocarnitine treatment } \\
\qquad(\mathrm{n}=9)\end{array}$ & $\begin{array}{c}\text { With } \\
\text { levocarnitine } \\
\text { treatment } \\
(\mathrm{n}=12)\end{array}$ & $\begin{array}{c}P \\
\text { values }\end{array}$ \\
\hline Age (year), median (IQR) & $67(63-75)$ & $76(66-79)$ & 0.0984 \\
\hline Sex (male/female) & $2 / 7$ & $1 / 11$ & 0.3681 \\
\hline $\begin{array}{l}\text { Etiology } \\
\text { (HCV/PBC/NASH/Alcohol }\end{array}$ & $4 / 0 / 3 / 2 / 0 / 0$ & $6 / 3 / 1 / 0 / 1 / 1$ & 0.7980 \\
\hline /HCV+PBC/HCV+HBV) & & & \\
\hline Child-Pugh class (A/B) & $6 / 3$ & $8 / 4$ & 1.0000 \\
\hline HCC yes/no (yes \%) & $1 / 8(11.1)$ & $2 / 10 \square 16.7 \square$ & 0.7188 \\
\hline 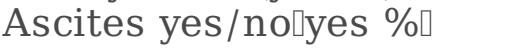 & $3 / 6(33.3)$ & 3/9ロ25.0॰ & 0.6757 \\
\hline Varices yes/nolyes \% & $2 / 7(22.2)$ & 2/7૧16.7૬ & 0.7483 \\
\hline Diabetes Mellitus & $4 / 5(44.4)$ & $2 / 10 \square 16.7 \square$ & 0.1632 \\
\hline yes/no (yes \%) & & & \\
\hline Shunt yes/no (yes \%) & 1/8 (11.1) & 2/10॰16.7॰ & 0.7188 \\
\hline $\begin{array}{l}\text { Levocarnitine dose } \\
1200 / 1800(\mathrm{mg})\end{array}$ & - & $6 / 6$ & \\
\hline
\end{tabular}


$\mathrm{HCV}$, hepatitis C virus; HBV, hepatitis B virus; $\mathrm{PBC}$, primary biliary cholangitis; NASH, non-alcoholic steatohepatitis; HCC, hepatocellular carcinoma; IQR, interquartile range. * $P$ $<0.05$ was considered significant.

Table 2 The comparison of laboratory data between baseline and after 3 months in cirrhotic patients with or without levocarnitine treatment. 


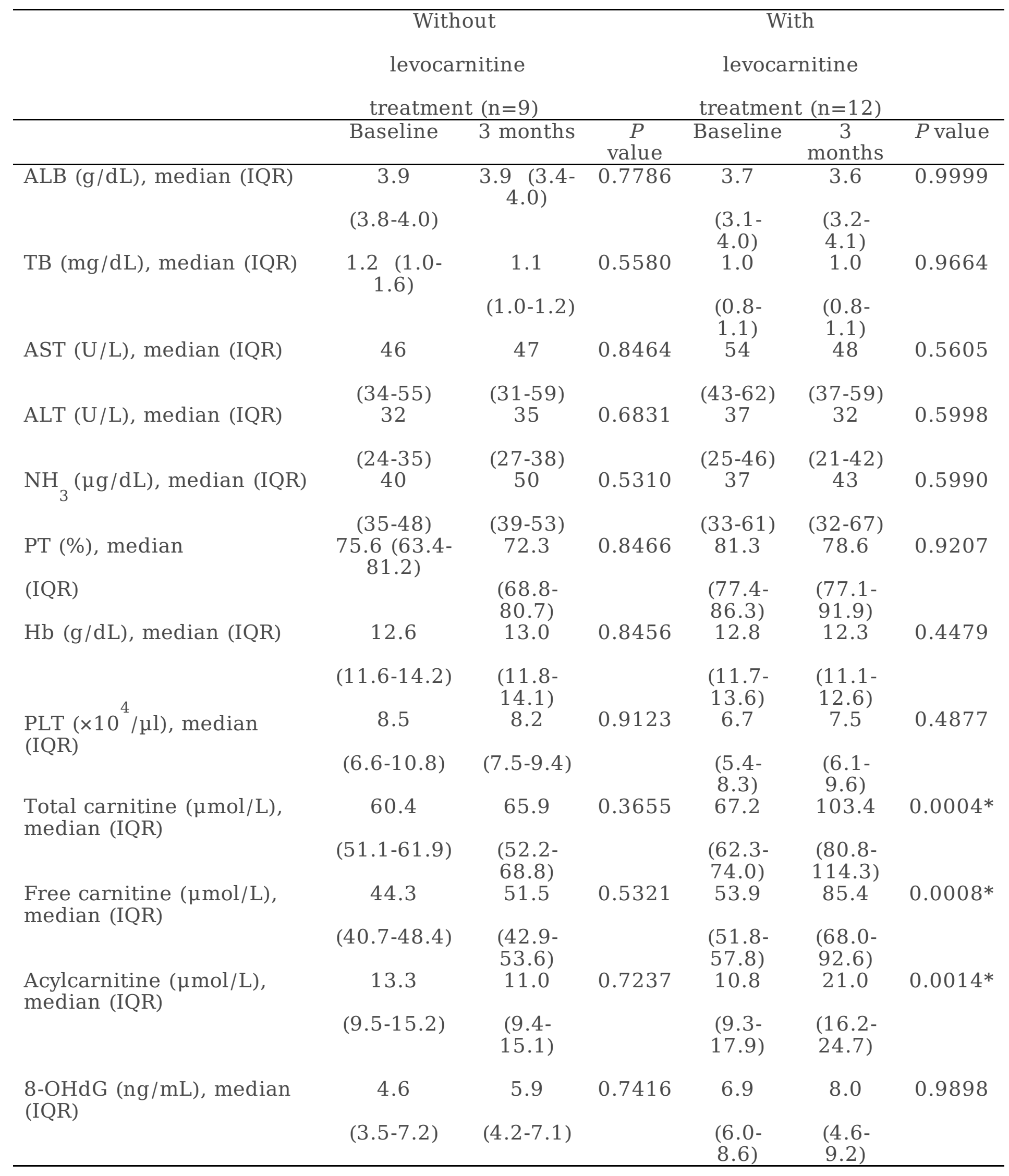

AST, aspartate aminotransferase; ALT, alanine aminotransferase; ALB, albumin; TB, total bilirubin; PT, prothrombin time; Hb, hemoglobin; PLT, platelet count; 8-OHdG, 8-hydroxy- 
2'-deoxyguanosine; IQR, interquartile range; ns, not significant. $* P<0.05$ was considered significant.

Table 3 Factors associated with the decreasing of serum thioredoxin levels after 3 months in cirrhotic patients without overt hepatic encephalopathy in multivariate analysis

\begin{tabular}{|c|c|c|c|c|c|}
\hline \multirow{2}{*}{$\begin{array}{c}\Delta \text { serum TRX levels of }<0 \mathrm{vs} \\
\geq 0\end{array}$} & & \multicolumn{2}{|c|}{ Model $1^{\mathrm{a}}$} & \multicolumn{2}{|c|}{ Model $2^{b}$} \\
\hline & & OR $(95 \%$ CI $)$ & $P$ & $\begin{array}{c}\mathrm{OR}(95 \% \\
\mathrm{CI}) \\
\end{array}$ & $P$ \\
\hline \multirow[t]{3}{*}{ FC concentration $(\mu \mathrm{mol} / \mathrm{L})$} & $>$ & $24.00(2.05-$ & $0.0112 *$ & 39.38 & $0.0186^{*}$ \\
\hline & & & & $\begin{array}{c}(1.85- \\
839.21)\end{array}$ & \\
\hline & $\begin{array}{l}\leq \\
61.5\end{array}$ & 1 (Ref) & & 1 (Ref) & \\
\hline $\mathrm{NH}_{3}$ & $<48$ & & & 3.71 & 0.3607 \\
\hline$(\mu \mathrm{g} / \mathrm{dL})$ & & & & $\begin{array}{l}(0.22- \\
61.67)\end{array}$ & \\
\hline & $\geq 48$ & & & 1 (Ref) & \\
\hline
\end{tabular}

Ref, reference group; OR, odds ratio; CI, confidence interval.

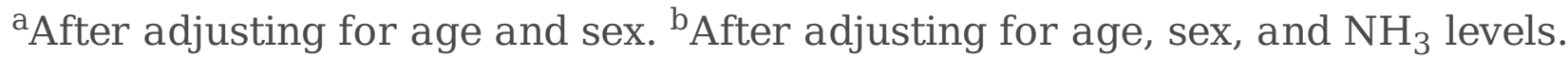

$\Delta$ serum TRX levels, [(serum TRX levels after 3months) - (serum TRX levels at baseline)]

$* P<0.05$ was considered significant.

Abbreviations: FC, free carnitine; TRX,thioredoxin.

\section{Figures}


Figure 1

Without levocarnitine treatment $(n=9)$

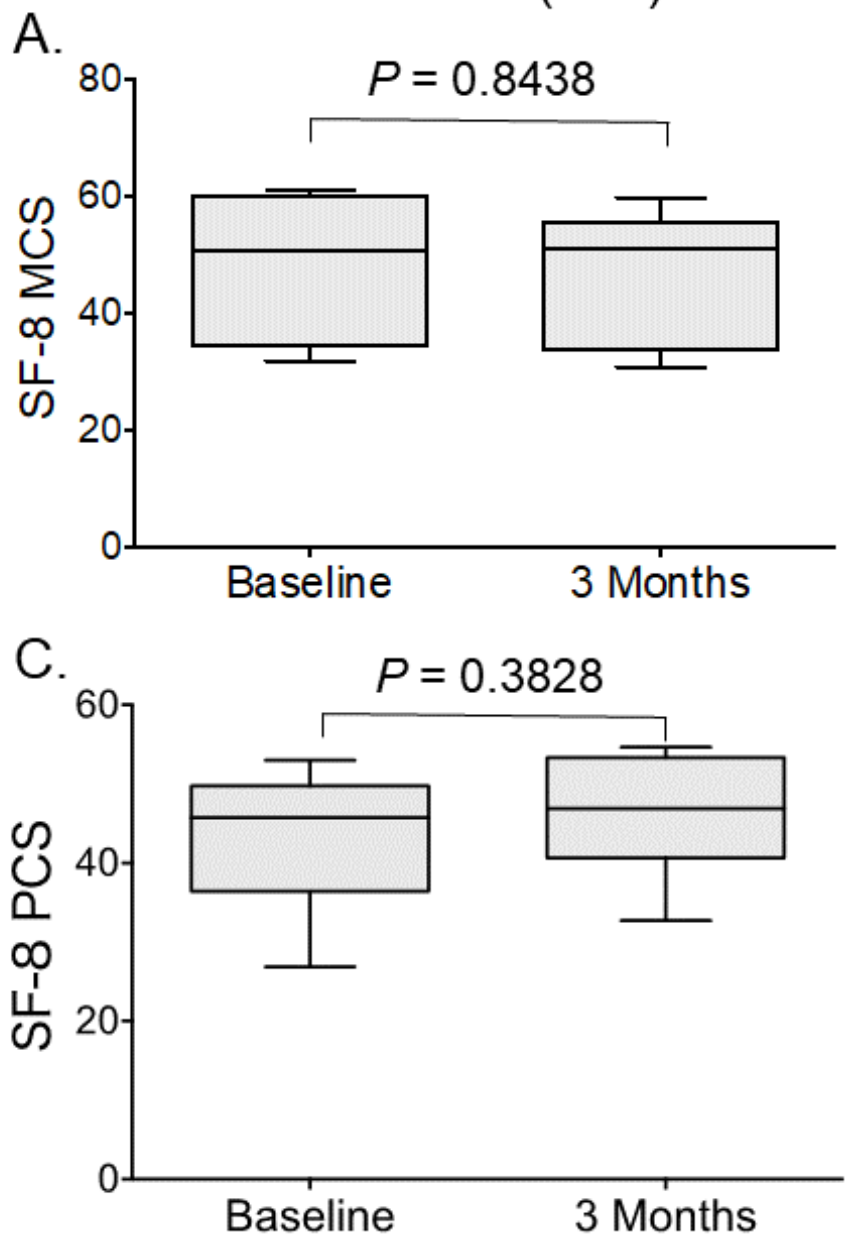

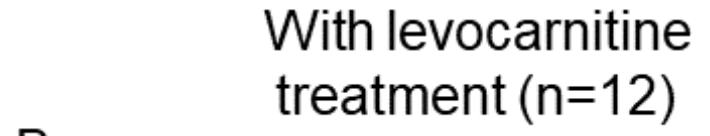

B.

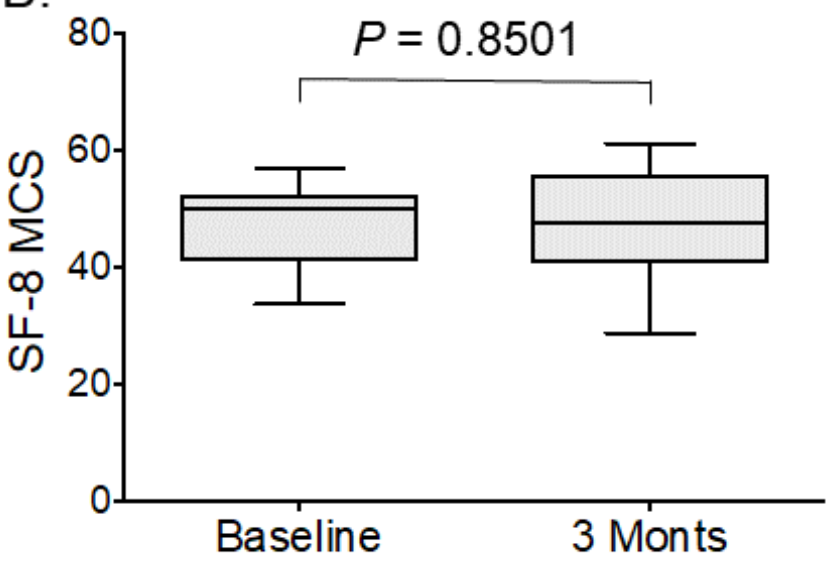

D.

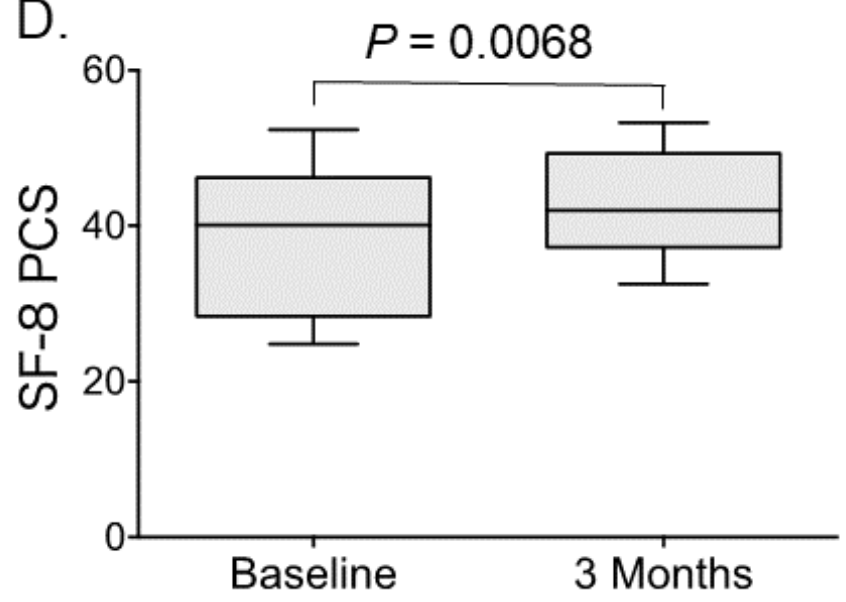

Figure 1

Results from the Japanese version of the Medical Outcomes Study Short-Form 8-Item Health Survey (SF8). (A) A comparison of the mental health component summary score (MCS) in cirrhotic patients who did not undergo levocarnitine treatment from baseline to 3 months after treatment. (B) A comparison of the MCS in cirrhotic patients who underwent levocarnitine treatment from baseline to 3 months after treatment. (C) A comparison of the physical health component summary score (PCS) in patients who did not undergo levocarnitine treatment from baseline to 3 months after treatment. (D) A comparison of the PCS in patients who underwent levocarnitine treatment from baseline to 3 months after treatment. $P<$ 0.05 indicated statistical significance. 
Figure 2

A. Without levocarnitine
treatment $(n=9)$

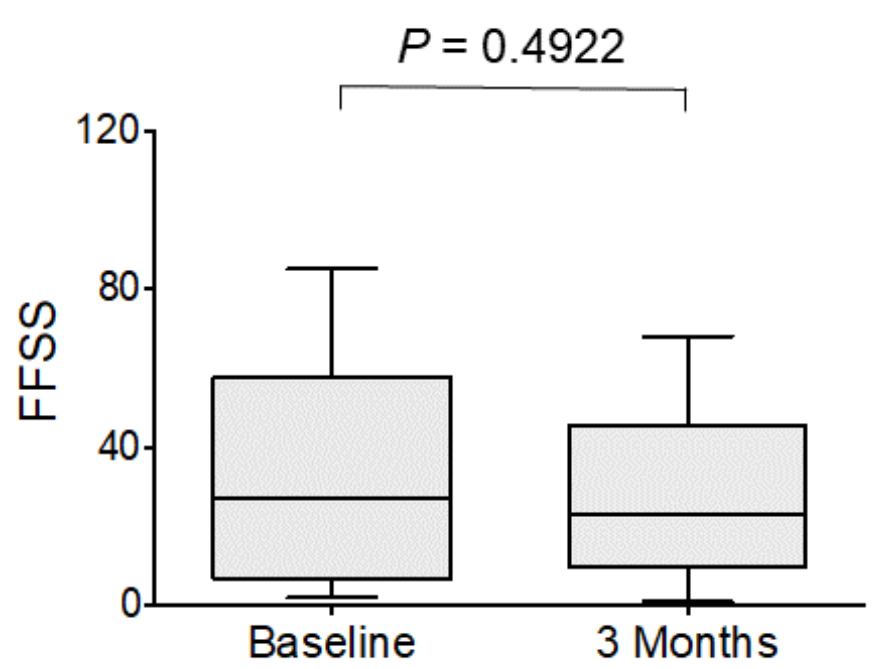

B. With levocarnitine treatment $(n=12)$

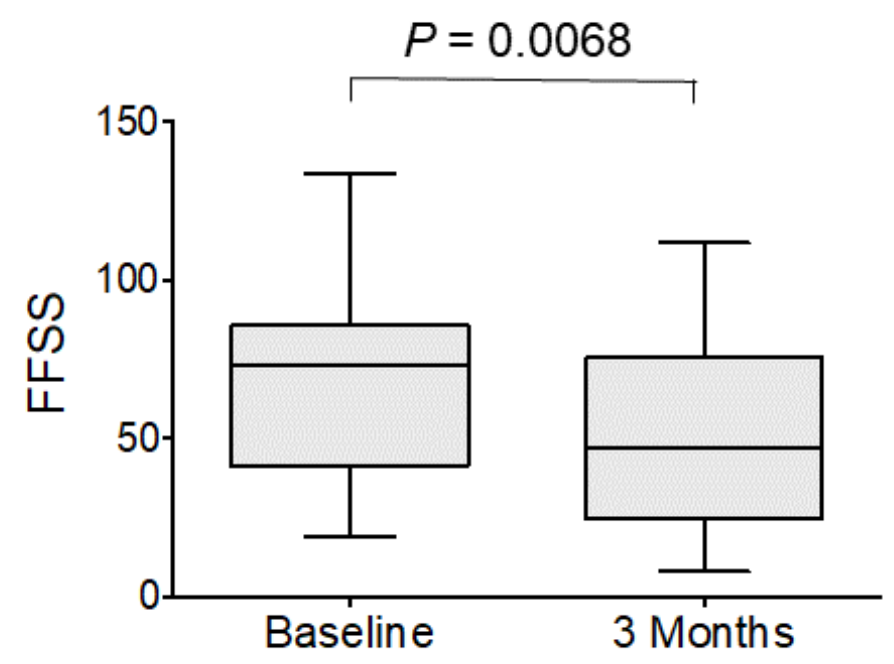

\section{Figure 2}

Comparison of the total score of the Japanese version of the Fisk Fatigue Severity Score (FFSS) in cirrhotic patients who did and did not undergo levocarnitine treatment. (A) A comparison of the FFSS in cirrhotic patients who did not undergo levocarnitine treatment from baseline to 3 months after treatment. (B) A comparison of the FFSS in cirrhotic patients who underwent levocarnitine treatment from baseline to 3 months after treatment. $P<0.05$ indicated statistical significance. 
A. Without levocarnitine treatment $(\mathrm{n}=9)$

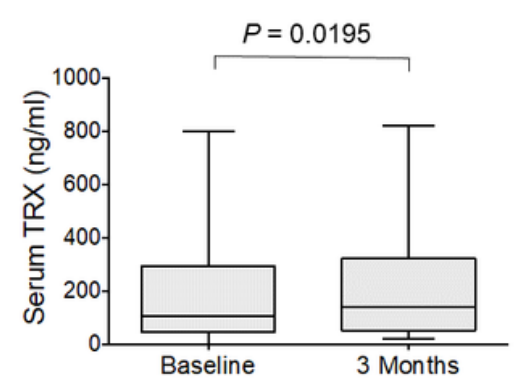

B. With levocarnitine treatment $(n=12)$

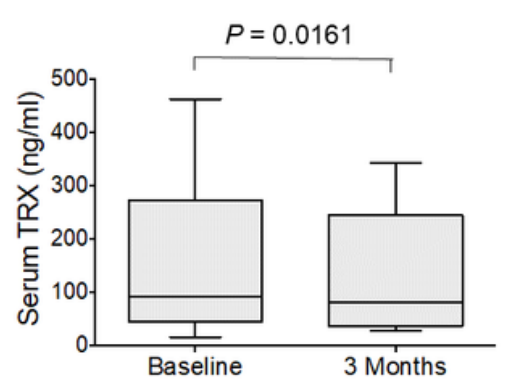

C.

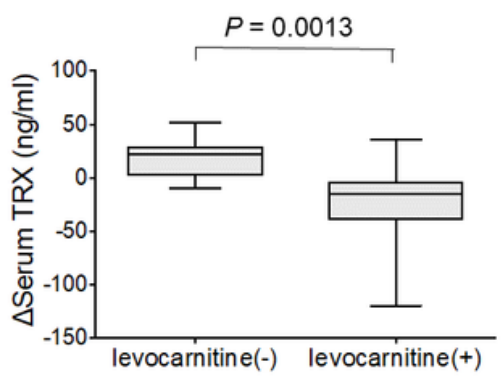

\section{Figure 3}

Serum thioredoxin levels in cirrhotic patients who did and did not undergo levocarnitine treatment. (A) A comparison of the serum thioredoxin (TRX) levels in cirrhotic patients who did not undergo levocarnitine treatment from baseline to 3 months later. (B) A comparison of the serum thioredoxin (TRX) levels in cirrhotic patients who underwent levocarnitine treatment from baseline to 3 months after treatment. (C) A comparison of the $\Delta$ serum TRX levels between cirrhotic patients who did and did not undergo levocarnitine treatment. $\mathrm{P}<0.05$ indicated statistical significance. 
Figure .4
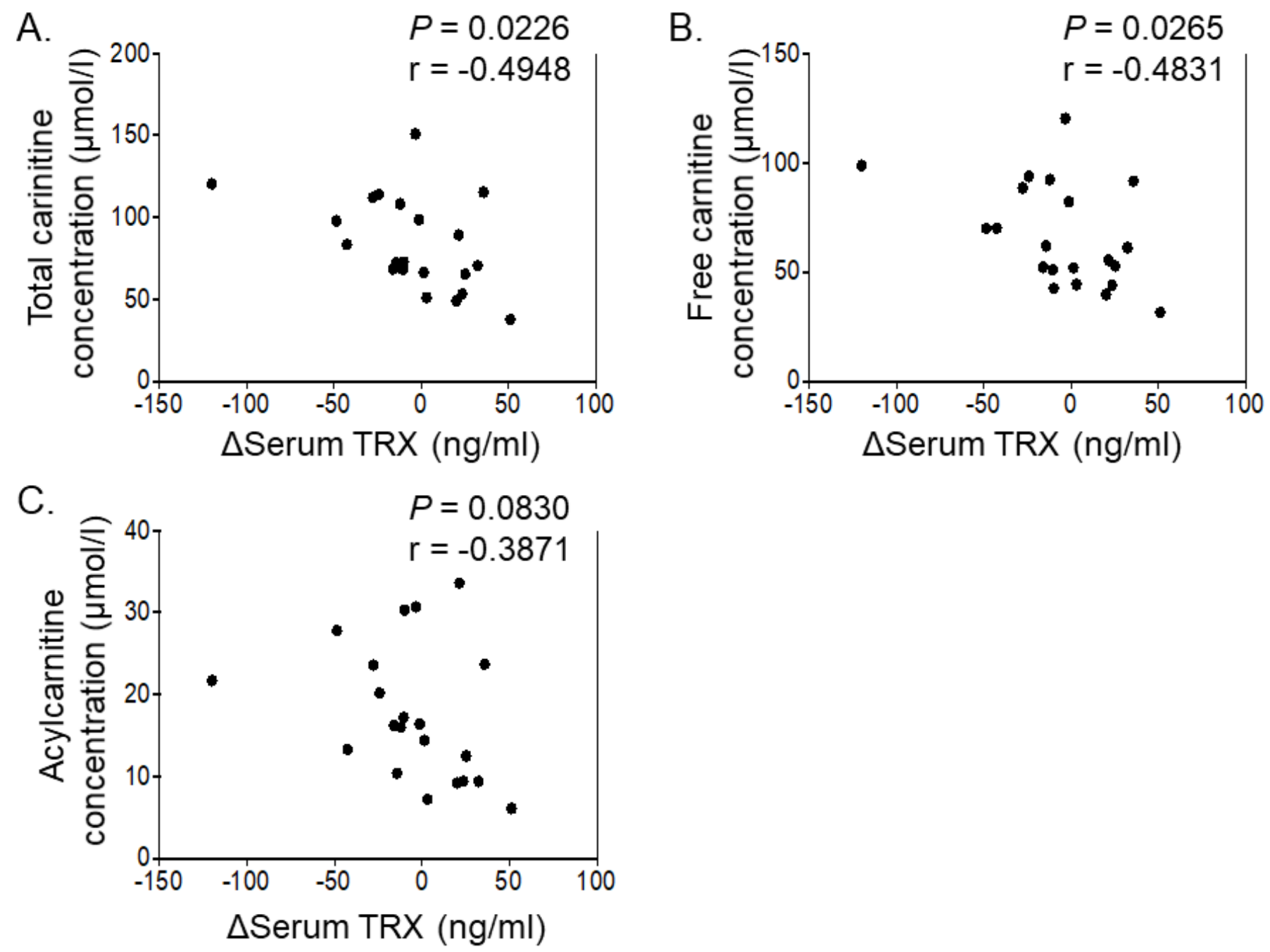

Figure 4

Relationship between serum carnitine fraction levels after 3 months and $\Delta$ serum thioredoxin levels in cirrhotic patients. (A) Relationship between serum total carnitine levels after 3 months and $\Delta$ serum thioredoxin (TRX) levels in cirrhotic patients. (B) Relationship between serum free carnitine levels after 3 months and $\Delta$ serum TRX levels in cirrhotic patients. (C) Relationship between serum acyl carnitine levels after 3 months and $\Delta$ serum TRX levels in cirrhotic patients. The $P$ values were calculated with the Spearman rank correlation test. $\mathrm{P}<0.05$ indicated statistical significance. 